\title{
Fungal Co-infections Associated with Global COVID-19 Pandemic: A Clinical and Diagnostic Perspective from China
}

\author{
Ge Song • Guanzhao Liang • Weida Liu
}

Received: 23 April 2020/Accepted: 25 May 2020/Published online: 31 July 2020

(C) Springer Nature B.V. 2020

\begin{abstract}
Coronavirus disease 2019 (COVID-19), caused by severe acute respiratory syndrome coronavirus 2 (SARS-CoV-2), has been sweeping across the globe. Based on a retrospective analysis of SARS and influenza data from China and worldwide, we surmise that the fungal co-infections associated with global COVID-19 might be missed or misdiagnosed. Although there are few publications, COVID-19 patients, especially severely ill or immunocompromised, have a higher probability of suffering from invasive mycoses. Aspergillus and Candida infections in COVID-19 patients will require early detection by a comprehensive diagnostic intervention (histopathology, direct microscopic examination, culture, $(1,3)-\beta$ D-glucan, galactomannan, and PCR-based assays) to ensure effective treatments. We suggest it is prudent to
\end{abstract}

Handling editor: Vishnu Chaturvedi.

Ge Song and Guanzhao Liang have contributed equally to this work.

G. Song · G. Liang · W. Liu ( $₫)$

Department of Medical Mycology, Institute of

Dermatology, Chinese Academy of Medical Science and

Peking Union Medical College \& Jiangsu Key Laboratory

of Molecular Biology for Skin Diseases and STIs,

Nanjing 210042, China

e-mail: liumyco@hotmail.com

W. Liu

Center for Global Health, School of Public Health,

Nanjing Medical University, Nanjing 211166, China assess the risk factors, the types of invasive mycosis, the strengths and limitations of diagnostic methods, clinical settings, and the need for standard or individualized treatment in COVID-19 patients. We provide a clinical flow diagram to assist the clinicians and laboratory experts in the management of aspergillosis, candidiasis, mucormycosis, or cryptococcosis as comorbidities in COVID-19 patients.

Keywords COVID-19 - SARS-CoV-2 - Fungal coinfection · Aspergillosis $\cdot$ Candidiasis

\section{The Global Popularity of COVID-19 and the Possibility of Fungal Co-infections}

As the human-to-human transmitted disease, coronavirus disease 2019 (COVID-19), caused by severe acute respiratory syndrome coronavirus 2 (SARSCoV-2), has been an emergency global public health events [1, 2]. Till May 18th, 2020, the COVID-19 has rapidly spread to 212 countries and caused nearly 5 million laboratory-confirmed cases and more than 310,000 deaths globally. Like SARS-CoV and Middle East Respiratory Syndrome Coronavirus (MERS$\mathrm{CoV}$ ), SARS-CoV-2 is responsible for lower respiratory infection and can cause Acute Respiratory Distress Syndromes (ARDS) [3]. Besides, the diffuse alveolar damage with severe inflammatory exudation, COVID-19 patients always have immunosuppression 
with a decrease in CD4 $+\mathrm{T}$ and CD8 $+\mathrm{T}$ cells [4]. Critically ill patients, especially the patients who were admitted to the intensive care unit (ICU) and required mechanical ventilation, or had a longer duration of hospital stays, even as long as 50 days, were more likely to develop fungal co-infections [5]. Hence, it is important to notice that COVID-19 patients can develop further fungal infections during the middle and latter stages of this disease, especially severely ill ones [6].

\section{Epidemiology of Fungal Co-infections in COVID- 19 Patients}

To analyze the incidence of fungal co-infections in COVID-19 patients, we searched PubMed, Scopus, Embase, and Web of Science, using the keywords "fungi" OR "fungus" OR "fungal infection" OR "invasive fungal diseases" OR "secondary infection" AND “COVID-19” OR “SARS-CoV-2” OR “2019nCoV" OR "2019 novel coronavirus" without date (up to May 18, 2020) and language restrictions. We also searched CNKI and Wanfang Data using the same terms in Chinese, with no time restrictions. The title, abstract, and full text of related articles determined according to these search criteria were carefully reviewed by the authors. Unfortunately, we have found very few articles reporting on fungal coinfections, not only that, some studies have not provided the details of the pathogens. Even so, we found COVID-19 patients, especially severely ill ones or accompanied with immunocompromised state, had co-infections of fungi [7]. In China, Chen et al. performed fungal culture on all 99 COVID-19 patients at admission and found five $(5 \%, 5 / 99)$ cases of fungal infection, including one case of Aspergillus flavus, one case of Candida glabrata and three cases of $C$. albicans [8]. Yang et al. found there (3/52, 5.8\%) patients had fungal co-infection in 52 critically ill patients, including A. flavus, A. fumigatus and $C$. albicans [5]. Other China studies have found a higher percentage of secondary infections $(8-15 \%)$ in COVID-19 patients, but it is not clear whether it is bacterial or fungal infection $[9,10]$. In addition, one study mentioned that $2.8 \%$ (31/1099) patients were treated with antifungal medicine, including $1.9 \%$ (18/ 926) non-severe patients and $7.5 \%$ (13/173) severe patients, but there was no etiological evidence of fungal co-infection [11]. Another study mentioned there was no patient treated with antifungal medicine in 149 cases [4]. A German study found COVID-19 associated invasive pulmonary aspergillosis (IPA) was found in five $(26.3 \%)$ of 19 consecutive critically ill patients with moderate to severe ARDS [12]. In Netherlands, there were six patients $(19.4 \%)$ presumed IPA in 31 ICU patients, of which five were identified A. fumigatus [13]. Besides, among the 5 first welldescribed French COVID-19 patients, an old severely ill man was co-infected with A. flavus by tracheal aspirates culture [14].

\section{Neglected Fungal Co-infection in COVID-19 Patients by Suggestive Ideas from SARS and Influenza}

Studies have shown that SARS-CoV and SARS-CoV2 belong to the same species and have the similar prevalence, biological and clinical characteristics [15]. Looking back on SARS in 2003, we found the incidence of fungal infection in SARS patients was $14.8-27 \%$, which was even higher in severely ill ones, up to $21.9-33 \%$ [16, 17], meanwhile, fungal infection was the main cause of death for SARS patients, accounting for $25-73.7 \%$ in all causes of death [18]. Besides, in the past decade, increasing reports of severe influenza pneumonia resulting in ARDS complicated by fungal infection were published [19]. One research found IPA was diagnosed in 83 (19\%) of 432 patients admitted with influenza, which was higher in immunocompromised patients (32\%), and in the event of IPA, the mortality will increase from 28 to $51 \%$ [20]. However, as for fungal co-infection in COVID19 patients, only few studies have reported it, which may have been neglected. Clinically, many COVID19 patients did not undergo sputum fungal assessment at the beginning, moreover, it is difficult to detect fungus with a single sputum fungal culture [11]. With the disease aggravating, it is easy to attribute the severe respiratory symptoms to COVID-19, at the most considering of the co-infection with bacterium or even mycoplasma [21] which usually leads to the intime use of antibiotics, while the diagnosis of fungal infection is always delayed or neglected. Based on the experience of SARS in 2003 and the cases of invasive aspergillosis combined with severe influenza, it is 
critically important to pay attention to the probability of COVID-19 accompanied by fungal infections.

\section{Clinical and Diagnostic Perspective of COVID-19 Associated with Fungal Co-infections}

As the ongoing COVID-19 pandemic, more and more experts are aware of fungal co-infections. The French High Council for Public Health recommended to systematically screen for fungal pathogens in COVID19 patients [6]. Lanjuan $\mathrm{Li}$ academician and her colleagues who have accumulated experience with severe COVID-19 treatment, reminded clinicians should focus on patients' fungal infections, especially severely ill or immunocompromised ones [22]. At the early phase of the disease or with extrapulmonary fungal infections, it may present with atypical chest imaging. Hence, it is necessary for severely ill patients to receive fungal pathogens surveillance, including (i) etiological examination: direct microscopy and culture; (ii) histopathology; (iii) serology: antigen and antibody, (1,3)- $\beta$-D-glucan (BDG) [23] and galactomannan (GM) detection by serum are also need to be tested for suspicious patients, while bronchoalveolar lavage fluid (BALF) and tracheal aspirate (TA) sampling for culture and biomarker testing should be performed under well-protected conditions due to the risk of aerosol spreading and health care worker infections [24]; (iv) PCR-based methods: Real-time polymerase chain reaction (PCR) techniques and molecular identification can be performed to identify pathogens if necessary [25]. After identifying the pathogen, the antifungal susceptibility testing (AST) can be performed to select sensitive antifungal drugs. If the AST cannot be carried out, it should be treated empirically. The main fungal pathogens for fungal coinfections in severe COVID-19 patients are Aspergillus and Candida. Other infrequent opportunistic pathogenic fungus caused lung infections also need to be considered, such as Mucor and Cryptococcus.

\section{Invasive Aspergillosis (IA)}

Aspergillus species could be an important cause of life-threatening infection in COVID-19 patients, especially in those with high risk factors. The potential risk factors for the patients include GC use, prolonged neutropenia, chronic obstructive pulmonary disease
(COPD), allogeneic hematopoietic stem cell transplant (allo-HSCT) [26], solid organ transplant (SOT) [27], inherited immunodeficiencies, hemopoietic malignancy (HM), cystic fibrosis (CF) [28], etc. The diagnosis of IA requires a microbiologic and/or histopathologic evidence, although specimen acquisition is challenging in many patients because lung biopsy might be contraindicated in patients with coagulation disorders or severe respiratory failure [13]. Histopathologic examination mainly rely on finding special fungal stains on lung fluid or tissue when a fungal infection is suspected and may reveal the characteristic acute angle branching septate hyphae of Aspergillus spp., and Grocott-Gomori's methenamine-silver stain (GMS) and periodic acidSchiff (PAS) stains of fixed tissue will helpful, while it is difficult to distinguish Aspergillus spp. from other filamentous fungi such as Fusarium spp. and Scedosporium spp. [29]. Therefore, it is necessary to have a definitive confirmation by culture or nonculture technique, including (i) direct microscopic examination with the optical brightener methods, Calcofluor or Blankophor, which may increase the sensitivity and specificity for detecting Aspergillus-like features; (ii) culture on fungal-specific media at $37{ }^{\circ} \mathrm{C}$ for 2-5 days, if positive, morphological features of Aspergillus can be identified under the microscope or the DNA sequencing may be used in reference laboratories to identify the species accurately, but usually culture yield is low and a negative result does not exclude the diagnosis of IA; (iii) molecular assays targeting ribosomal DNA (rDNA) sequences can also be used for detection of Aspergillus in tissues or BALF, especially PCR-based assays can be used to detect Aspergillus spp. and CYP51A resistance mutations in A. fumigatus, although these methods have not been standardized or limited by laboratory conditions or proven commercial reagents in some countries [30]; (iv) serum and BALF GM testing are also recommended as an early and accurate marker using less invasive techniques for the diagnosis, especially in neutropenic patients, with advantages of less injury and time-efficient, though sometimes this test in blood samples are less sensitive than cultures of respiratory samples [25].

The treatment recommendations can be supported by the 2016 Update guideline by the Infectious Diseases Society of America that the prophylaxis, therapeutic medication, combined, and alternative 
medication of aspergillus infection have been given more detailed guidance opinions [30]. Generally, drugs recommended for the treatment and prophylaxis of IA include triazoles (itraconazole, voriconazole, posaconazole, esaconazole), Amphotericin B and its liposomes and echinococcins (micafungin or carpofenjing). Most patients can choose triazole drugs to treat IA, however, therapeutic drug monitoring (TDM) is recommended and the interaction between azoles and other drugs should be fully considered.

Invasive Candidiasis (IC)

For the severe COVID-19 patients who have more opportunities to be treat with broad-spectrum antibacterial drugs, parenteral nutrition and invasive examinations, or the patients accompanied with prolonged neutropenia and other immune impairment factors, the risk of infection with Candida species may significantly increase [31]. Diagnosis of IC depends on culture methods including culture of blood or other samples collected under sterile conditions which are usually considered as gold standards for IC, and nonculture diagnostic tests including mannan and antimannan IgG tests, C. albicans germ tube antibody (CAGTA), BDG and PCR-based assays, which are now entering clinical practice as adjuncts to cultures [32]. There are mainly two disadvantages about blood culture, on the one hand, the blood culture time is long, because the average positive alarm time is 2-3 days (range 1 to $\geq 7$ days), plus identification and susceptibility test duration 4 to 6 days, on the other hand, it is not sensitive than PCR with much lower detection limit when Candida concentration is $\leq 1 \mathrm{CFU} / \mathrm{mL}$ and easy to have failure to detect in extremely low concentrations of candidiasis, intermittent candidiasis or deep Candida infection has not entered the blood. Hence, several nonculture diagnostic tests are recommended, but also there is widespread uncertainty about their utility in clinical practice [31]. BDG is a major cell wall constituent of Candida and most pathogenic fungi, excluding Cryptococcus species, Blastomyces species, and Mucorales, which is widely used in clinical and well recommended by detecting serum, but cannot distinguish between Candida and other fungi [25]. Besides, mannan and antimannan IgG tests, CAGTA are employed at many European centers, and higher sensitivity and specificity by a combination with mannan/antimannan assay are observed [33].
There are promising PCR tests, including multiplexPCR platforms, at the same time, it exists some limitations for a lack of multicenter validation of assay performance, so there are no FDA-cleared PCR assays for Candida, but commercial and in-house tests are widely available. Further, T2 magnetic resonance is also can be used by amplifying and detecting Candida DNA, but its feasibility in early diagnosis of candidemia remains unclear. MALDI-TOF technology is available in more hospitals with the biggest advantage of its promptness taking no more than $5 \mathrm{~min}$ to identify a microorganism from isolated colonies, even researchers have developed protocols to identify yeasts directly from positive blood culture bottles within half an hour without performing a subculture [32]. Overall, not only it is necessary to fully realize the benefits of combining culture and nonculture methods, but also, clinicians must take the types of IC, the strengths and limitations of each assay and the context of the clinical setting into account to have a judicious interpretation. Besides, susceptibility test is recommended for all blood-stream and other clinically relevant Candida isolates, especially for C. glabrata or C. parapsilosis.

The treatment recommendations can be supported by the 2016 Update guideline by the Infectious Diseases Society of America that the therapeutic and alternative medication of candidiasis infection have been given more detailed guidance opinions [34]. Generally, patients who are suspected or confirmed with IC should be treated with echinocandin (caspofungin, micafungin, and anidulafungin), azoles (fluconazole, voriconazole, itraconazole), and Amphotericin B and its liposomes, what's more, TDM for azoles should be used to optimize efficacy and limit toxicity.

Invasive Mucormycosis

COVID-19 patients with trauma, diabetes mellitus, GC use, HM, prolonged neutropenia, allo-HSCT, SOT are more likely to develop mucormycosis [35]. Mucormycosis is usually suspected based on results of direct microscopy or plus fluorescent brighteners from clinical specimens such as sputum, BALF, and skin lesions that Mucorales hyphae are non-septate or pauci-septatethe with a variable width of 6-16 $\mu \mathrm{m}$. To confirm the diagnosis, non-pigmented hyphae showing tissue invasion should be shown in tissue sections 
stained with hematoxylin-eosin (HE), PAS, or GMS [36]. Culture of specimens is strongly recommended for identification of genus and species, also AST. What's more, it is suggested to be cultured at $30{ }^{\circ} \mathrm{C}$ and $37{ }^{\circ} \mathrm{C}$ separately that typically cottony white or grayish black colony usually will be found, afterward morphological identification of fungi or DNA sequencing based on bar code genes, such as $18 \mathrm{~S}$, ITS, $28 \mathrm{~s}$, or rDNA. MALDI-TOF identification is just moderately supported because it depends mainly on in-house databases, and many laboratories do not have this capacity [37]. Further, it is promising to detect fungi DNA, in serum as well as in other body fluids, even in paraffin-embedded tissue, however, because of lack of standardization supported it is only with moderate strength.

The treatment recommendations can be supported by the global guideline for the diagnosis and management of mucormycosis in 2019 by European Confederation of Medical Mycology (ECMM) and Mycoses Study Group Education and Research Consortium that the therapeutic and alternative medication of mucormycosis have been given more detailed guidance opinions [35]. Generally, it strongly supports an early complete surgical treatment for mucormycosis whenever possible, in addition to systemic antifungal treatment. In neutropenic patients, those with graftversus-host disease or high risk factor, primary prophylaxis with posaconazole may be recommended. Amphotericin B lipid complex, liposomal Amphotericin $\mathrm{B}$ and posaconazole oral suspension are treated as the first-line antifungal monotherapy, while isavuconazole is strongly supported as salvage treatment. There are no convincing data to guide the use of antifungal combination therapy of polyenes and azoles or polyenes plus echinocandins.

\section{Invasive Cryptococcosis}

COVID-19 patients with human immunodeficiency virus (HIV) infection accompanied by $\mathrm{CD} 4+\mathrm{T}$ lymphocyte count $<200$ cells $/ \mu \mathrm{L}$, allo-HSCT, SOT, or other immune impaired are susceptible to cryptococcosis which predominantly present as meningoencephalitis [38]. Given the complexities surrounding the diagnosis of cryptococcosis and identification of Cryptococcus species including C. neoformans and $C$. gattii species, the diagnosis of cryptococcosis is usually based on a combination of clinical and laboratory confirmation. The methods used to confirm the infection are culture, direct microscopy, histopathology, serology, and molecular detection. To diagnose cryptococcosis, specimen from cerebrospinal fluid (CSF) can be mixed with India ink and observed under a microscope that the distinctive structure for Cryptococcus spp. with narrow budding encapsulated yeasts usually can be found. Samples for culture should be placed on Sabouraud dextrose agar at $30{ }^{\circ} \mathrm{C}$ for 7 days, in aerobic conditions, and observed daily. Moreover, cultures from patients receiving systemic antifungal therapy might need longer to grow. Cryptococcus appears as mucoid creamy colonies. Capsular polysaccharides of Cryptococcus can be detected and quantified from body fluids such as serum, CSF, BAL, or pathological tissue. Three formats of cryptococcal antigen (CrAg) detection tests are currently available: the latex agglutination test (LAT), the enzyme-linked immunoassay (EIA), and the lateral flow immunoassay (LFA). These methods are rapid, sensitive, and specific, but have not been standardized for respiratory specimens such as BAL, pleural fluid, or sputum [32]. Molecular detection of Cryptococcus is required in specific situations where other diagnostic tools have failed to confirm a diagnosis of cryptococcosis. These molecular methods include pan-fungal PCR, DNA sequencing for identification, multiplex PCR, isothermal amplification method, and probe-based microarrays. Once a diagnosis cryptococcosis is made, a lumbar puncture and cerebrospinal fluid (CSF) examination (including antigen) are recommended in all patients [39]. Cryptococcus can disseminate into the central nervous system causing cryptococcal meningitis.

The treatment recommendations can be supported by guidelines for the diagnosis, prevention, and management of cryptococcal disease in HIV-infected adults, adolescents, and children in 2018 by World Health Organization (from: https://www.who.int/hiv/ pub/guidelines/cryptococcal-disease/en/). Generally, the following is recommended as the preferred regimen: (i) Induction phase for amphotericin B deoxycholate and + flucytosine, followed by fluconazole; alternative options for fluconazole + flucytosine or amphotericin B deoxycholate + fluconazole. (ii) Consolidation phase for fluconazole. (iii) Maintenance (or secondary prophylaxis) phase for fluconazole. 


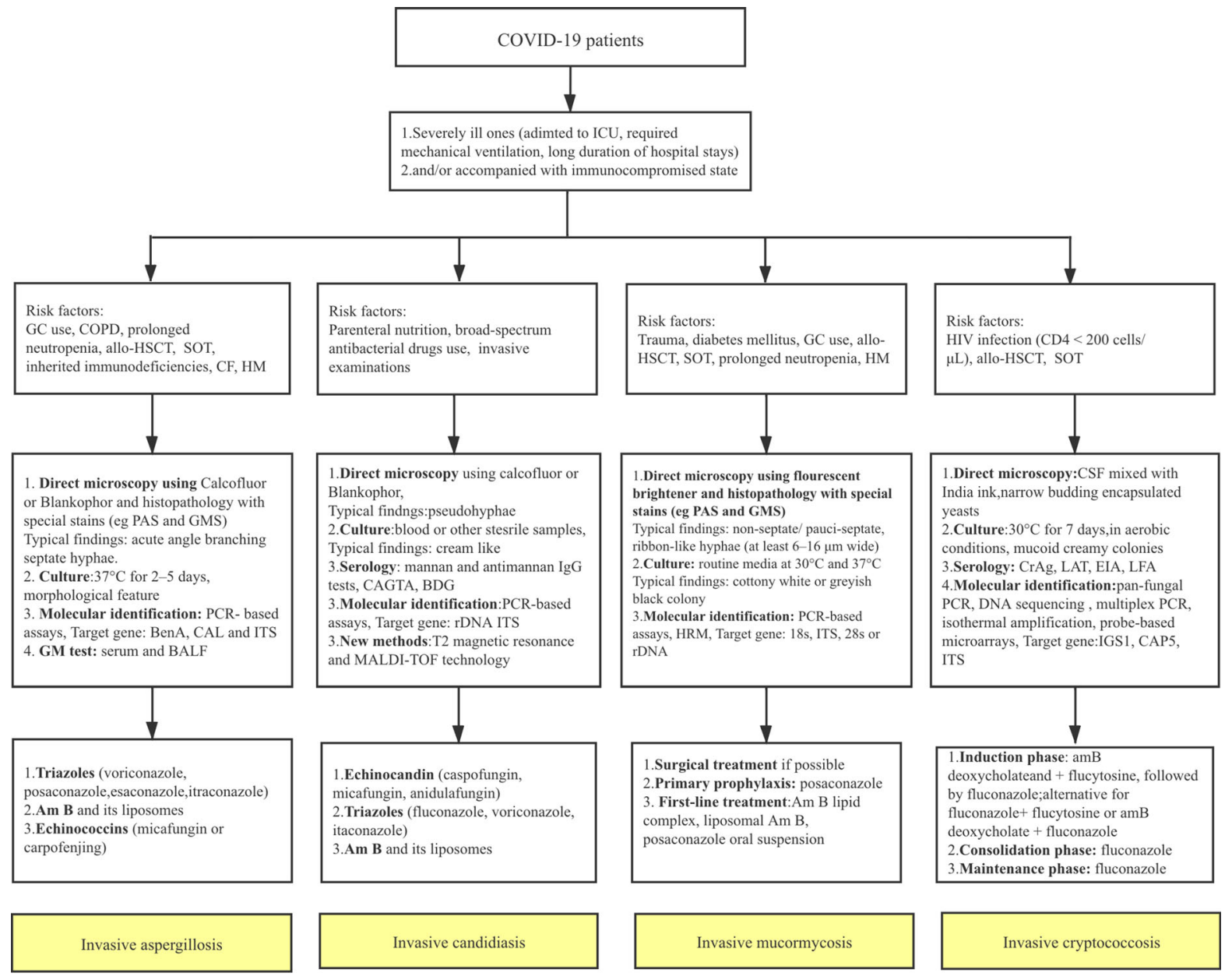

Fig. 1 Diagnostic and therapeutic pathway for invasive fungal co-infection

\section{Summary}

By analyzing retrospective analysis of SARS and influenza data from China and worldwide, we surmise that the fungal co-infections associated with global COVID-19 might be missed or misdiagnosed. Further, as a life-threatening infectious disease, COVID-19 patients showed overexpression of inflammatory cytokines, and impaired cell-mediated immune response with decreased CD $4+\mathrm{T}$ and $\mathrm{CD} 8+\mathrm{T}$ cell counts, indicating its susceptibility to fungal coinfection. Moreover, COVID-19 patients accompanied with immunocompromised state, such as prolonged neutropenia, HSCT, GC use, SOT, inherited or acquired immunodeficiencies, and tumor are more likely to develop fungal co-infection. Here, we summarized updated diagnostic information (histopathology, direct microscopic examination, culture, (1,3)- $\beta$-D-glucan, galactomannan, PCR-based assays, MALDI-TOF technology, etc.) and treatment recommendations of invasive mycosis. We suggest it is prudent to assess the risk factors, the types of invasive mycosis, the strengths and limitations of diagnostic methods, clinical settings, and the need for standard or individualized treatment in COVID-19 patients. Finally, provide a clinical flow diagram (Fig. 1) to assist the clinicians and laboratory experts in the management of aspergillosis, candidiasis, mucormycosis, or cryptococcosis as comorbidities in COVID-19 patients.

Funding This work was supported by The subitem of the Important and Special Project of the Science and Technology Ministry of China (No. 2018ZX10734404) and National Pathogen Resource Center. 


\section{Compliance with Ethical Standards}

Conflict of interest The authors declare that they have no conflict of interest.

\section{References}

1. Zhou P, Yang XL, Wang XG, Hu B, Zhang L, Zhang W, et al. A pneumonia outbreak associated with a new coronavirus of probable bat origin. Nature. 2020;579(7798): 270-3.

2. Gorbalenya AE, Baker SC, Baric RS, et al. The species severe acute respiratory syndrome-related coronavirus: classifying 2019-nCoV and naming it SARS-CoV-2. Nat Microbiol. 2020;5(4):536-44.

3. Wang Y, Wang Y, Chen Y, Qin Q. Unique epidemiological and clinical features of the emerging 2019 novel coronavirus pneumonia (COVID-19) implicate special control measures. J Med Virol. 2020. https://doi.org/10.1002/jmv. 25748.

4. Yang W, Cao Q, Qin L, Wang X, Cheng Z, Pan A, et al. Clinical characteristics and imaging manifestations of the 2019 novel coronavirus disease (COVID-19): a multi-center study in Wenzhou city, Zhejiang, China. J Infect. 2020. https://doi.org/10.1016/j.jinf.2020.02.016.

5. Yang X, Yu Y, Xu J, Shu H, Xia J, Liu H, et al. Clinical course and outcomes of critically ill patients with SARSCoV-2 pneumonia in Wuhan, China: a single-centered, retrospective, observational study. Lancet Respir Med. 2020. https://doi.org/10.1016/s2213-2600(20)30079-5.

6. Gangneux JP, Bougnoux ME, Dannaoui E, Cornet M, Zahar JR. Invasive fungal diseases during COVID-19: we should be prepared. J Mycol Med. 2020. https://doi.org/10.1016/j. mycmed.2020.100971.

7. Guo L, Wei D, Zhang X, Wu Y, Li Q, Zhou M, et al. Clinical features predicting mortality risk in patients with viral pneumonia: the MuLBSTA score. Front Microbiol. 2019; $10: 2752$.

8. Chen N, Zhou M, Dong X, Qu J, Gong F, Han Y, et al. Epidemiological and clinical characteristics of 99 cases of 2019 novel coronavirus pneumonia in Wuhan, China: a descriptive study. Lancet. 2020;395(10223):507-13.

9. Huang C, Wang Y, Li X, Ren L, Zhao J, Hu Y, et al. Clinical features of patients infected with 2019 novel coronavirus in Wuhan, China. Lancet. 2020;395(10223):497-506.

10. Ruan Q, Yang K, Wang W, Jiang L, Song J. Clinical predictors of mortality due to COVID-19 based on an analysis of data of 150 patients from Wuhan, China. Intensive Care Med. 2020. https://doi.org/10.1007/s00134-020-05991-x.

11. Guan WJ, Ni ZY, Hu Y, Liang WH, Ou CQ, He JX, et al. Clinical characteristics of coronavirus disease 2019 in China. N Engl J Med. 2020. https://doi.org/10.1056/ NEJMoa2002032.

12. Koehler P, Cornely OA, Bottiger BW, Dusse F, Eichenauer DA, Fuchs F, et al. COVID-19 associated pulmonary aspergillosis. Mycoses. 2020. https://doi.org/10.1111/myc. 13096.

13. van Arkel ALE, Rijpstra TA, Belderbos HNA, van Wijngaarden $P$, Verweij PE, Bentvelsen RG. COVID-19 associated pulmonary aspergillosis. Am J Respir Crit Care Med. 2020. https://doi.org/10.1164/rccm.202004-1038le.

14. Lescure FX, Bouadma L, Nguyen D, Parisey M, Wicky PH, Behillil S, et al. Clinical and virological data of the first cases of COVID-19 in Europe: a case series. Lancet Infect Dis. 2020. https://doi.org/10.1016/s1473-3099(20)30200-0.

15. Peeri NC, Shrestha N, Rahman MS, Zaki R, Tan Z, Bibi S, et al. The SARS, MERS and novel coronavirus (COVID-19) epidemics, the newest and biggest global health threats: what lessons have we learned? Int J Epidemiol. 2020. https://doi.org/10.1093/ije/dyaa033.

16. Zhang Y, Li WX, Huang KW, Cao ZX, Hao JY. Hospital acquired pneumonia occurring after acute stage of the serious SARS and its treating strategies. Chin J Nosocomiol. 2003;11(13):1081-7.

17. Yin $\mathrm{CH}$, Wang C, Tang Z, Zhang SW, Wang BS. Clinical analysis of 146 patients with critical severe acute respiratory syndrome in Beijing areas. Clin J Emerg Med. 2004;1(13): $12-4$.

18. Li CS, Pan SF. Analysis and causation discussion of 185 severe acute respiratory syndrome dead cases. Zhongguo wei zhong bing ji jiu yi xue. 2003;15(10):582-4.

19. Thevissen K, Jacobs C, Holtappels M, Toda M, Verweij P, Wauters J. International survey on influenza-associated pulmonary aspergillosis (IAPA) in intensive care units: responses suggest low awareness and potential underdiagnosis outside Europe. Crit Care. 2020;24(1):84.

20. Schauwvlieghe A, Rijnders BJA, Philips N, Verwijs R, Vanderbeke L, Van Tienen C, et al. Invasive aspergillosis in patients admitted to the intensive care unit with severe influenza: a retrospective cohort study. Lancet Respir Med. 2018;6(10):782-92.

21. Chaturvedi V, Bouchara JP, Hagen F, Alastruey-Izquierdo A, Badali H, Bocca AL, et al. Eighty years of mycopathologia: a retrospective analysis of progress made in understanding human and animal fungal pathogens. Mycopathologia. 2018;183(6):859-77.

22. Xu K, Cai H, Shen Y, Ni Q, Chen Y, Hu S, et al. Management of corona virus disease-19 (COVID-19): the Zhejiang experience. Zhejiang da xue xue bao Yi xue ban. 2020; 49(1):0.

23. Lahmer T, da Costa CP, Held J, Rasch S, Ehmer U, Schmid $\mathrm{RM}$, et al. Usefulness of 1,3 beta-D-glucan detection in nonHIV immunocompromised mechanical ventilated critically ill patients with ARDS and suspected Pneumocystis jirovecii pneumonia. Mycopathologia. 2017;182(7-8):701-8.

24. Prattes J, Valentin T, Hoenigl M, Talakic E, Reisinger AC, Eller P. Invasive pulmonary aspergillosis complicating COVID-19 in the ICU-a case report. Med Mycol Case Rep. 2020. https://doi.org/10.1016/j.mmcr.2020.05.001.

25. Hage Chadi A, Carmona Eva M, Epelbaum Oleg, Evans Scott E, Gabe Luke M, Haydour Qusay, Knox Kenneth S, Kolls Jay K, Hassan Murad M, Wengenack Nancy L, Limper Andrew H. Erratum: Microbiological laboratory testing in the diagnosis of fungal infections in pulmonary and critical care practice. An Official American Thoracic Society Clinical Practice Guideline. Am J Respir Crit Care Med. 2019;200(10):1326.

26. Miceli MH, Churay T, Braun T, Kauffman CA, Couriel DR. Risk factors and outcomes of invasive fungal infections in 
allogeneic hematopoietic cell transplant recipients. Mycopathologia. 2017;182(5-6):495-504.

27. Fishman JA, Grossi PA. Novel Coronavirus-19 (COVID19) in the immunocompromised transplant recipient: \#Flatteningthecurve. Am J Transplant. 2020. https://doi.org/ 10.1111/ajt.15890.

28. Poli P, Timpano S, Goffredo M, Padoan R, Badolato R. Asymptomatic case of Covid-19 in an infant with cystic fibrosis. J Cyst Fibros. 2020. https://doi.org/10.1016/j.jcf. 2020.03.017.

29. Blot SI, Taccone FS, Van den Abeele AM, Bulpa P, Meersseman W, Brusselaers N, et al. A clinical algorithm to diagnose invasive pulmonary aspergillosis in critically ill patients. Am J Respir Crit Care Med. 2012;186(1):56-64.

30. Patterson TF, Thompson GR 3rd, Denning DW, Fishman JA, Hadley S, Herbrecht R, et al. Practice guidelines for the diagnosis and management of aspergillosis: 2016 update by the Infectious Diseases Society of America. Clin Infect Dis. 2016;63(4):e1-60.

31. Clancy CJ, Nguyen MH. Diagnosing invasive candidiasis. J Clin Microbiol. 2018;56(5):e01909-17.

32. Ibanez-Martinez E, Ruiz-Gaitan A, Peman-Garcia J. Update on the diagnosis of invasive fungal infection. Rev Esp Quimioter. 2017;30(Suppl 1):16-21.

33. Mikulska M, Calandra T, Sanguinetti M, Poulain D, Viscoli C. The use of mannan antigen and anti-mannan antibodies in the diagnosis of invasive candidiasis: recommendations from the Third European Conference on Infections in Leukemia. Crit Care. 2010;14(6):R222.
34. Pappas PG, Kauffman CA, Andes DR, Clancy CJ, Marr KA, Ostrosky-Zeichner L, et al. Clinical practice guideline for the management of candidiasis: 2016 update by the Infectious Diseases Society of America. Clin Infect Dis. 2016;62(4):e1-50.

35. Cornely OA, Alastruey-Izquierdo A, Arenz D, Chen SCA, Dannaoui E, Hochhegger B, et al. Global guideline for the diagnosis and management of mucormycosis: an initiative of the European Confederation of Medical Mycology in cooperation with the Mycoses Study Group Education and Research Consortium. Lancet Infect Dis. 2019;19(12): e405-21.

36. Skiada A, Lass-Floerl C, Klimko N, Ibrahim A, Roilides E, Petrikkos G. Challenges in the diagnosis and treatment of mucormycosis. Med Mycol. 2018;56(suppl 1):93-101.

37. Dadwal SS, Kontoyiannis DP. Recent advances in the molecular diagnosis of mucormycosis. Expert Rev Mol Diagn. 2018;18(10):845-54.

38. Setianingrum F, Rautemaa-Richardson R, Denning DW. Pulmonary cryptococcosis: a review of pathobiology and clinical aspects. Med Mycol. 2019;57(2):133-50.

39. Gassiep I, Douglas J, Emeto TI, Crawley K, Playford EG. Cryptococcal infections over a 15 year period at a tertiary facility \& impact of guideline management. Mycoses. 2018;61(9):633-8.

Publisher's Note Springer Nature remains neutral with regard to jurisdictional claims in published maps and institutional affiliations. 